Aus der dermatologischen Universitätsklinik in Leipzig.

(Direktor Prof. Riehl.)

\title{
Über Ulcus molle miliare, sogenannten Follikularschanker.
}

\author{
Von \\ Dr. Hans Vörner, \\ Assistent an der Klinik für Dermatologie und Syphilis zu Leipzig.
}

Das Auftreten entzündlicher Knötchen am Genitale, deren Ätiologie dem Virus des Ulcus molle zugeschrieben wird und deren Symptome einer Folliculitis gleichkommen, von sogenanntem Follicularschanker, ist kein seltenes Ereignis.

Trotzdem fehlen bisher genauere, ätiologisch beweisende, namentlich histologische Untersuchungen dieser Affektion, deren Bezeichnung, Ulcus molle folliculare, selbst nach der klinischen Seite nicht ganz einwandsfrei ist.

Bei der ersten Untersuchung von Kranken findet man vielfach neben einem typischen Ulcus molle eine beschränktere oder größere Zahl von "follikularen Knötchen". Beobachtet man die Individuen fortgesetzt, so können mitunter einige dieser Effloreszenzen spontan heilen, andere gehen in typische weiche Geschwüre über. An anderen Patienten dagegen sieht man anfänglich eine Anzahl von entzündlichen Knötchen bestehen, die sich dann früher oder später in ulcera mollia umwandeln.

Wie überall auf dem Derma, wo Follikel existieren, besteht auch am Genitale natürlich gelegentlich eine Folliculitis simplex beziehentlich eine Acne vulgaris. ${ }^{1}$ ) Es ist also die Deutung bei Fällen der ersten Art möglich, daß es hiebei zu einer Autoinfektion gekommen ist, indem von dem schon bestehenden Geschwür infektiöses Material in die gleich-

1) Petersen, Ulcus molle I. Teil. Archiv für Dermatologie und Syphilis Bd. XXX. p. 381. 
zeitig oder später entstandenen Folliculitiden gelangt sei. Bei entsprechender Entwicklung des Schankergittes würde dann aus dieser Effloreszenz ein Ulcus molle entstehen. Im anderen Falle könnte eine schon bestehende Akne durch Kontagion mit Ulcus molle-Bazillen infiziert sein oder die betreffenden Knötchen würden einer gleichzeitigen Verimpfung von Eitererregern und Streptobazillen ibre Entstehung verdanken können. (Mischinfektion).

Schließlich spricht die Entstehungsweise des Ulcus molle, besonders bei der experimentellen Überimpfung anscheinend nicht dafür, daß der Keim des Ulcus molle auch im stande ist, eine Folliculitis zu erzeugen. Denn wir sehen bei Anwendung des sonst üblichen Verfahrens stets primär eine Pustel auftreten. ${ }^{\mathbf{1}}$ )

Diese Beobachtungen und Schlüße haben mich veranlaßt, die folgenden Untersuchungen auszuführen und, da ihre Ergebnisse mir von gewisser Bedeutung schienen, kurz mitzuteilen.

Schon längst hatte ich an den Patienten auf der Geschlechtsstation des Krankenhauses zu St. Jakob die Beobachtung gemacht, daß diejenigen Knötchen an den Genitalien, aus welchen man später Ulcera mollia sich entwickeln sah, gegenüber den Effloreszenzen einer einfachen, durch Eitererreger hervorgerufenen Folliculitis sich nach gewisser Richtung hin anders verhielten. Sie zeigten eine intensivere und weiter reichende, auf Druck verschwindende Rötung ihrer Umgebung, die ziemlich reichlich über den Umfung des Knötchens hinausreichte; weiterhin eine frühzeitige, tief in die Cutis reichende, nur wenig rezistent sich anfühlende Infiltration. Auf der Spitze des Knötchens kam es nicht zu einer sich vorwölbenden Eiterpustel, sondern es bildete sich an der Spitze des Knötchens ein feinstes, deutlich eingezogenes Eiterpünktchen, das nach Wegtupfen des Sekretes schon eher den Eindruck eines kleinen, die obersten Hautschichten durchbohrenden, mit Eiter gefüllten Loches machte. Manchmal hatte dasselbe seinen Sitz in einem Haartrichter, so daß das Haar direkt durch den Eiterpunkt hindurch-

1) Die Natur des weichen Schankers. XI. internationaler medizinischer Kongreß in Rom. Monatshefte für praktische Dermatologie Bd. XIX. 1894. II. Theil. 
ging, oder in der öffnung eines Drüsenausführungsganges. Vielfach indessen konnte man eiue derartige Lokalisation nicht nachweisen, sondern das Knötchen stieg frei aus der Cutis empor. Die Farbe des Eiters hat immer einen ausgesprochen zitronengelben Ton, ziemlich gesättigt und feuchtglänzend. Das Lnnötchen bildet anfänglich einen kleinen spitzen Kegel. Sobald sich das Eiterpünktchen entwickelt, wird nit der Ausdehnung desselben auch die Spitze des Knötchens niedriger, sodaß dasselbe einem mehr und mehr abgestumpften Kegel ähnlich wird. Gleichzeitig vertieft sich das Eiterpünktchen, wird kraterförmig. Waihrend die Umgebung sich abflacht, wird aus demselben allmählich ein scharfrandiges Geschwür, so daß bei Hanfkorngröße schon alle Symptome des Ulcus molle vorhanden sein können.

Die Schankerknötchen widerstehen sehr oft den sonst für die Behandlung des Ulcus moile üblichen Methoden. ${ }^{1}$ ) Dic größte Sicherheit des Erfolges bietet die Excision dieser Efflorescenzen und die nachträgliche Verschorfung der Wunde (z. B. mit Acidum carbolicum). Auf diese Weise waren meine Patienten früher von ihrem Leiden und seinen eventuellen recht unangenehmen Folgen (vor allem Bubo) befreit und ich erwarb zur histologischen Lntersuchung verwertbare Objekte. 1)ieselben stammen fast alle von weiblichen Individuen, die eine weit größßere Neigung, an diesen Kffloreszenzen zu erkranken, besitzen, als männliche.

Drei Knötchen, welche die oben geschilderten charakteristischen Symptome des "Follikularschankers" in den ersten Anfängen erkennen ließen, im besonderen, an deneu der zentrale Eitcrpunkt im frühesten Entstehen beziehentlich eben sichtbar. ward, wurien in Alkohol gehärtet, in Paraffin gebettet, dann in Serien und zwar in senkrechter Richtung zur Oberfläche geschnitten.

Das erste dieser in Serien greschnittenen Knötchen war vom großen Iabium excidiert worden. Bei der Durchsicht der Schnitte zeigte es sich, daß das makroskopisch sichtbare Knötchen durch Finlagerung von Zellmassen in Herdform in dor Papilarschicht und Cutis verursacht war Dieser Herd hob der Gestalt des Knötchens entsprechend die üher demselben befindliche Fipidermis über das Nivean der gesunden Umgebung. Nach abwärts begrenzte sich das Knötchen bogenförmig, so daß der

1) Lesser, Geschlechtskrankheiten. 1901, pag. 82. 
Herd in seiner Ausdebnung ziemlich einer Kugel entsprach. Der größte Durehmesser betrug zirka $11 / 2 \mathrm{~mm}$.

Die Epidermis über dem Knötchen zeigt eine deutliche Quellung der Fpithel- und Hornzellen, die Interstitialräume zwischen den Zellen ind von zablreichen Eiter- beziehen tlich Wanderzellen durchsetzt. Die Retezapfen der über dem Knötchen befindlichen Retepartie etwas verflacht, dagegen am Rande gegen das Gesunde zu mehr verdickt.

Auf Schnitten, welche mehr oder weniger die Mittelachse des Knötchens treffen, sieht man, daß Hornschicht und Rete malpighii von einem feinem Kanal durchbohrt werden, der ganz mit Eiterzellen erfüllt ist. Im größten Durchmesser seines Lumens hat der Porus zirka 0.1 $\mathrm{mm}$. Die die Wand des Kanals bildenden Epidermiszellen sind etwas aufgeloekert, die denselben erfüllende Eitersäule setzt sich direkt in den subepidermoidalen Herd fort.

Der Herd selbst besteht in überwiegender Weise aus bezüglich des Kern stark sich tingierenden polynucleären Eiterzellen, namentlich gegen die Peripherie zu finden sich auch Plasmazellen, verzweigte oder einzelne spindelförmige Zellen mit blaßem größeren Kern, gelockerte Bindegewebsfibrillen und stark erweiterte, meist von Eiterzellen erfüllte Lymphgefäße.

Im Eiterkanal der Epidermis bis fast in die Mitte des Entzündungsherdes hinein gewahrt man schmale Bazil'en von sehr geringer Gröbe, die der von U $\mathrm{n}$ a und anderen für den Streptobazillus des Ulcus molle angegebenen entspricht, die in der Längsachse hintereinander liegend zu langen Ketten vereinigt sind. Dieselben beobachtet man teils eínzeln, teils zu Bündeln vereinigt, welche sich gelegentlich wieder in einzelne Ketten anflösen können. Die Ketten winden sich in Schlangenlinien oder Spiraltouren durch die Masse der Eiterzellen. Die Bazillen särben sich gut mit polychromem Methylenblau nach $\mathrm{Unna}_{\mathrm{a}}{ }^{1}$ ) mit Thionin nach $\mathrm{Nicolle}^{\mathbf{2}}$ ) und entfärben nach Gram.

Der Porus des Eiterherdes öffnet sich nach außen im äußersten Trichter eines Haarfollikels. Bei Durchsicht sämtlicher Schnitte erkennt man, daß das Haar den Knoten nieht im Zentrum durebbohrt, sondern nur in stark schräger Weise einen kleinen äußeren Anteil desselben. Der Entzündungsherd hat sich in seiner Hauptmasse seitlich vom Haarbalg entwickelt. Im Innern des Haarkanales sowie der zugehörigen Talgdrüse ist nirgend eine Eiterzellenanhäufung zu sehen. Nur insoweit Haarbalg and Haartrichter vom Entzündungsherd berührt werden, finden sich zwischen den Epithelien einzelne Eiterzellen, im übrigen ist Haarbalg und die zugehörige Talgdrüse völlig verschont.

Die Blutgefäße in der Umgebung des Knötchens sind sämtlich stark

1) Unna, Der Streptobazillus des weichen Schankers, Monatsheft für praktische Dermatologie 1892, I. Teil, XIV. Bd., p. 485.

2) Maurice Nicolle, Methode générale de coloration des bacilles qui ne prenent point le Gram. Annales de l'Instituts Pasteur, novembre 1892. 
in ihrem Durchmesser vergrößert und von reichlichen Zellmengen umgeben. In gleicher Weise sind die Lymphgefäße stark, spaltenartig erweitert.

Von den beiden anderen Knötchen stammte das eine aus der Schenkelfalte, das andere vom kleinen Labium. In diesen Fällen war genau derselbe Befund zu konstatieren, nur befanden sich diesmal die Herde in gar keiner Beziehung zu einem Haartrichter oder zu der Öffnung einer Talgdrüse, sondern dieselben saßen direkt unter der vorgewölbten Epidermis und ibr Porus mündete an der freien Oberfläche der Haut.

Das bemerkenswerte an diesem Befunde besteht vor allem in dem bisher noch nicht geführten Nachweise des Vorhandenseins der Streptobazillen in diesen Effloreszenzen. Die schon eingangs erwäbnten Entstehungsmöglichkeiten, die man dem klinischen Bilde nach wohl in Erwägung ziehen mußte, treffen also nicht zu Besonders handelt es sich nicht um eine Mischiufektion mit Eitererregern, welcher ihrer Natur nach die Entstehung von entzündlichen, dann vereiternden Knötchen wohl zuzuschreiben wäre. Der Streptobazillus ist allein in Reinkultur innerhalb dieser Effloreszenzen zu finden.

Weiterhin kommen aber für diese Fälle auch nicht Knötchen oder Bläschen in Betracht, welche sekundär infiziert wurden, denn dafür fehlen tatsächliche Anhaltspunkte. Vor allem widerspricht die Bezeichnung mit dem Namen eines Follıkularschankers $d$. h. eines durch den Erreger des Ulcus molle hervorgerufenen Folliculitis, dem oligen Befunde. Unter Folliculitis verstehen wir eine primäre Entzündung der Haar- oder Talgfollikel, in unseren Fällen nimmt die Affektion nicht einmal ihren Ausgangspunkt von diesen Organen und dieselbenwerden höchstens sekundär in Mitleidenschaft gezogen.

Das anatomische Substrat unserer Effloreszenzen ist vielmehr ein durch den Streptobazillus hervorgerufener kleinster entzündlicher Herd, welcher, wie das größere typische Ulcus molle, allerdings in den kleinsten Dimensionen eine Perforation mit lotrechten Wänden durch die Epidermis verursacht und in dic Cutis ein entzündliches Infiltrat mit den gleichen charakteristischen Eigenschaften veranlaßt. Auffallend wäre nur die Knötchenform, welche durch das empordrängende Infiltrat bei noch fest zu*ammenhängender aber nachgiebiger Epidermis über demselben zu stande kommt. Indessen haben wir nur bei fehlender Epidermis ein ganz ähnliches Verhalten des 
Geschwürgrundes nur in noch stärker ausgesprochenem Maße in dem nicht seltenen Ulcus molle elevatum.

Es wäre demnach nicht unpassend, den Namen Follikularschanker fallen zu lassen und die Effloreszenzen als Ulcus molle miliare $\mathrm{zu}$ bezeichnen.

Bei der Durchsicht weiterer Präparate, allerdings nicht in Serien habe ich meine obigen Ausfübrungen nur bestätigt gefunden.

Warum der Streptobazillus einmal ein Ulcus molle, ein andermal ein miliares Geschwür hervorruft, läßt sich natürlich nicht ohne weiteres beantworten. Vielleicht spielen folgende Momente eine gewis.e Rolle.

Impfen wir experimentell eine Hautstelle mit dem Sekret des Ulcus molle, so skarifizieren wir gewöhnlich eine zirka $1 \mathrm{~cm}$ große Stelle z. B. nach $\mathrm{K}$ reft in $\mathrm{g}^{1}$ ) mit drei Strichen einer Impflanzette. Auf die Weise bringen wir eine verhältnismäßig große Menge von spezifischem Material in die Haut und wir erhalten als Resultat eine größere Pustel, die nach Verlust ihrer Decke sofort zum Geschwür sich umwandelt. Gegenüber dieser Methode habe ich mit einer ganz dünnen, spitzen Mikroskopiernadel rersucht, Ulcus molle Belig, der mit steriler physiologischer Kochsalzlösung verdüunt war, unter Einhaltung aller aseptischen Kautelen, durch einen einzelnen ganz feinen Einstich in die Haut zu übertragen. Tatsächlich habe ich. unter vielen vergeblichen Experimenten doch zweimal miliare Ulcera angehen sehen.

Weiterhin fällt die Betrachtung von Schnitten, die typischen ulcera mollia angehören auf, daß die Streptobazillen meistens in zahlreichen Ketten und dichten Bündeln in der Hauptsache horizontal der Ebene der Cutis entsprechend entwickelt sind. Am miliaren Ulcus molle dagegen sehen wir die Bazillen nur an einer sehr kleinen umschriebenen Stelle zur Entwicklung gelangt und im wesentlichen in senkrechter Richtung. nach abwärts ziehen. Es ist nicht unmöglich, daß diese beschränkte Bazillenmenge und ihre Wachstumsrichtung dazu beitragen, daf es zur Entwicklung der Knötchenform komnit. Es läßt sich vermuten, daß die Vermehrung der Keime sowie ihre Ausbreitung in seitlicher Richtung dann die Umwandlung in ein typisches Ulcus molle zur Folge haben würde.

1) Krefting. Über die für Uleus molle specifische Mikrobe. Archiv für Dermatologie und Syphilis. Supplement 1892. p. 41. 